\title{
Diffusive two-dimensional spreading of a polymer
}

\author{
Uwe Albrecht, Achim Otto and Paul Leiderer \\ Fakultät für Physik, Unicersität Konstanz, D-7750 Konstanz, Germany
}

Received 20 April 1992; accepted for publication 12 June 1992

We have measured the thickness profiles of ultrasmall (picoliter volume) droplets of polydimethylsıloxane during their spreading on a silver surface at central thicknesses below one monolayer. The resulting profile shapes can be approximated well by shperical caps, and the diffusion dynamics are quite different from those expected for pointlike particles. The experimentill data are in remarkable agrcement with Monte Carlo simulations of a spreading two-dimensional polymer lattice-gats, which are performed using the bond flucluation method.

The fundamental physical concepts involved in the spreading of liquids on solid surfaces have been a topic of increasing interest in the past years. Following a detailed theoretical analysis by de Gennes [1], there have been a number of experiments probing the shapes and dynamics of liquid droplets spreading on a solid substrate [2]. So far the experiments as well as subsequent theoretical work [3] have focussed on the behavior of the central cap of the droplet and on the general properties of the precursor film that precedes the edge of the spreading cap. Our aim was to investigate the processes at the edge of the precursor film, where simple diffusive mechanisms are expected to dominate. However, detailed and systematic studies of this region have been lacking so far. We thus studied the temporal cvolution of droplet profiles with central thicknesses below one monolayer, using droplet volumes of only a few picoliters. The results are compared to Monte Carlo simulations of a spreading two-dimensional polymer gas.

We chose to study the spreading of polydimethylsiloxane (PDMS), since this polymer has been widely used in experiments by other groups as well. PDMS molecules lie flat on a solid surface and have a chain diameter of about $7 \AA$ [4]. They are commercially available with a large range of molecular weights. We worked with low weight, unfractionated polymers [5] in order to reduce the time scale of the experiments. The droplet profiles were measured using optically excited surface plasmons. Details of the experimental technique are given elsewhere [6]. The substrate consisted of a thin silver film (thickness $53 \mathrm{~nm}$ ), which was prepared by thermal evaporation onto the base of a glass prism. We deposited the PDMS shortly after preparation of the films. The experiments were performed under air in a closed box to avoid contamination by dust. The lateral resolution of the results shown below is about $120 \mu \mathrm{m}$.

Fig. 1 shows droplet profiles obtained during the spreading of a PDMS droplet with a $6 \mathrm{p} \ell$ volume. The earliest profile has a central thickness still larger than a monolayer. A small kink in the slopes indicates monolayer completion between 7 and $8 \AA$. The slight asymmetry is a result of the noticeable spreading while scanning the profile at this early stage. All later profiles have a central thickness of less than a monolayer. At these stages the droplet should be viewed as a two-dimensional distribution of polymers, accordingly the thickness profile should be more correctly labelled an areal density distribution (nevertheless, we will use the terms interchangeably). Assuming simple diffusive dynamics of pointlike particles, one would expect the profiles to have 


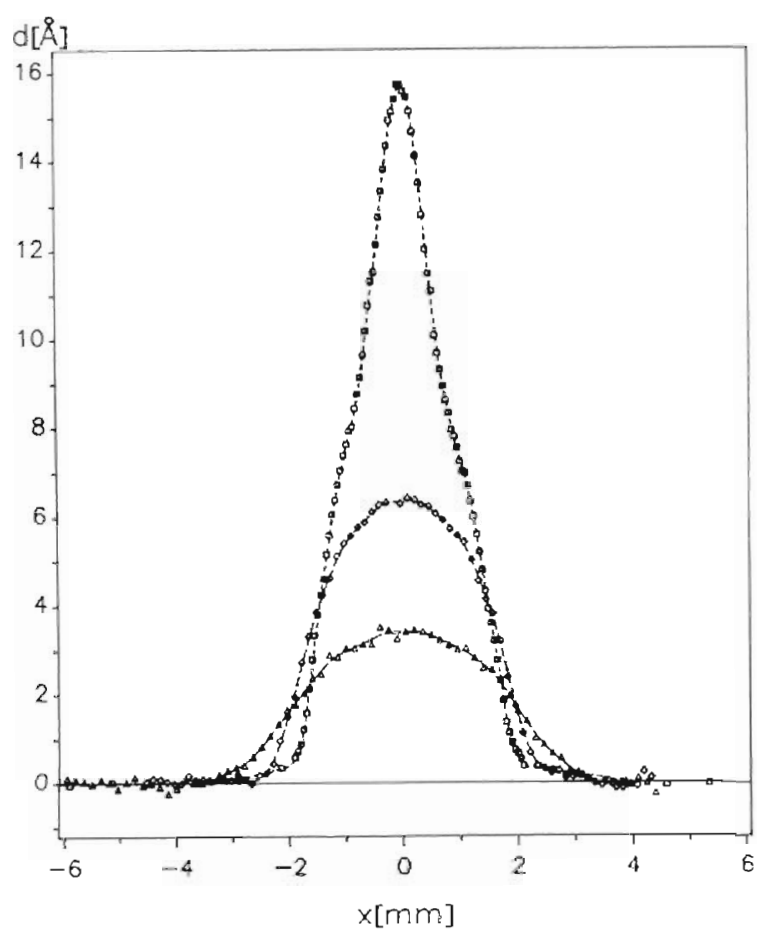

Fig. 1. Measured thickness profiles of a spreading PDMS droplet (volume $6 \mathrm{p} t$ ), at $t \equiv 35(\square), 80(\diamond)$ and $360(\Delta)$ min after deposition.

Gaussian shapes. However, fig. 2 shows that this is not the case. Fits of a Gaussian to one of the profiles in fig. 1 cannot satisfactorily describe the droplet shape, whereas it can be approximated

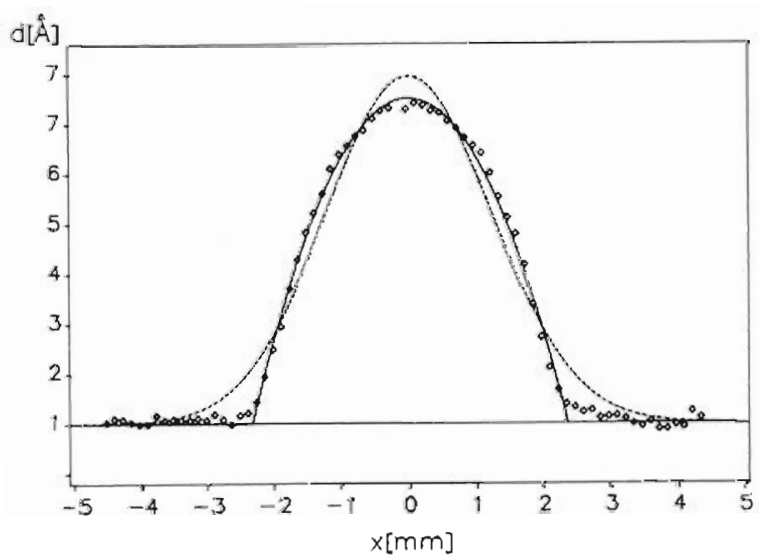

Fig. 2. Middle profile of fig. 1 fitted by a Gaussian (dashed) and a spherical cap (solid). very well by a spherical cap. This holds for all measured droplets. The small smeared out foot at the edge of the spherical cap, just visible in fig. 2 , grows larger with increasing spreading and will be discussed below. A description of the growth of the droplet radius $r$ by a simple growth law $r \sim t^{\gamma}$ yields exponents around 0.13 , considerably lower than the value of 0.5 expected for simple diffusion.

This result shows, that obviously a submonolayer of polymer molecules cannot be viewed as an ensemble of frecly diffusing particles. Like in three dimensions the polymer motion is restricted at high areal densities in the presence of other molecules, possibly leading to a reptation-like motion. Only at densities where the mean distance between the polymers is larger than their average extension free diffusion is expected to take over.

In order to check these qualitative arguments we have performed Monte Carlo simulations on a two-dimensional polymer lattice-gas employing an algorithm based on the bond fluctuation method introduced by Carmesin and Kremer [7]. Polymers are modelled as chains of monomers on a square lattice. They can move via hopping of single monomers, where the bond lengths are allowed to fluctuate. We used a single-site model (i.e., the bond length can have values of 1 and $\sqrt{2}$ in units of the lattice constant) on a $600 \times 600$ lattice, allowing only one monomer per lattice-site at any time. The initial areal density distribution was taken constant within a circle of radius 50 , located at the center of the lattice (this "pancake" shaped distribution is the final stage of a spreading droplet in the model of de Gennes [1], neglecting diffusion). The starting polymer configuration was achieved by growing the polymers in a self-avoiding way from an even distribution of lattice sites, allowing only a limited number of growth events. This process. quite analogous to real polymer growth, yields a natural distribution of polymer lengths. We allowed only a bond length of 1 during the growth to exclude blocked configurations (this restriction on the starting bond length has no influence on the results). Fig. 3 shows the polymer length distribution for the simulation results shown below. It was chosen to 


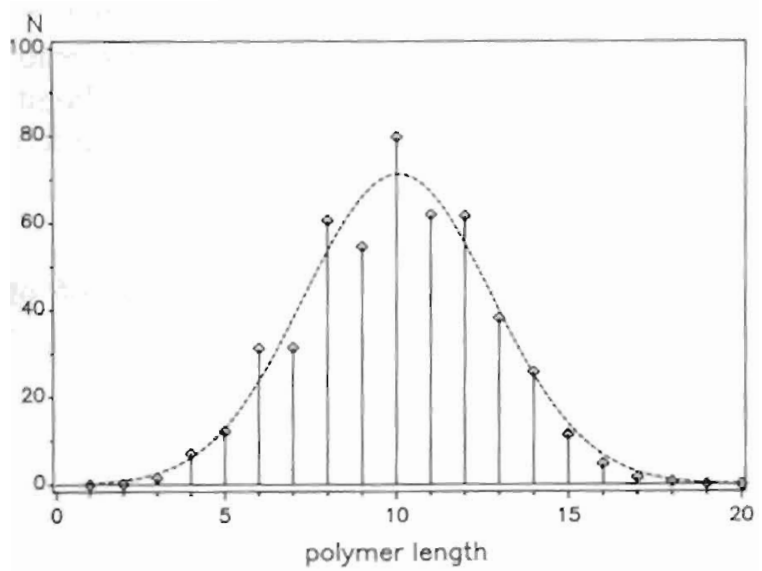

Fig. 3. Distribution of polymer lengths (in number of monomers) for the simulations shown in fig. 4 . match the polymer length used in the experiments, i.e., the average number of monomers agrees with the length in lattice constants.

The resulting profile shapes are displayed in fig. $4 \mathrm{a}$ at simulation times corresponding to the spreading time in the experiments shown in fig. $4 \mathrm{~b}$. The similarity between the simulated and the measured profiles is quite striking. Even the growth of the smeared out foot shows up in the same way in the simulation as in the experimental data. The profiles in fig. 4 have been fitted with spherical caps convoluted with a narrow Gaussian to account for the foot. The width of this Gaussian grows approximately with $t^{1 / 2}$, leading to the interpretation that it reflects the onset of free diffusion at the low density edges of the profiles.
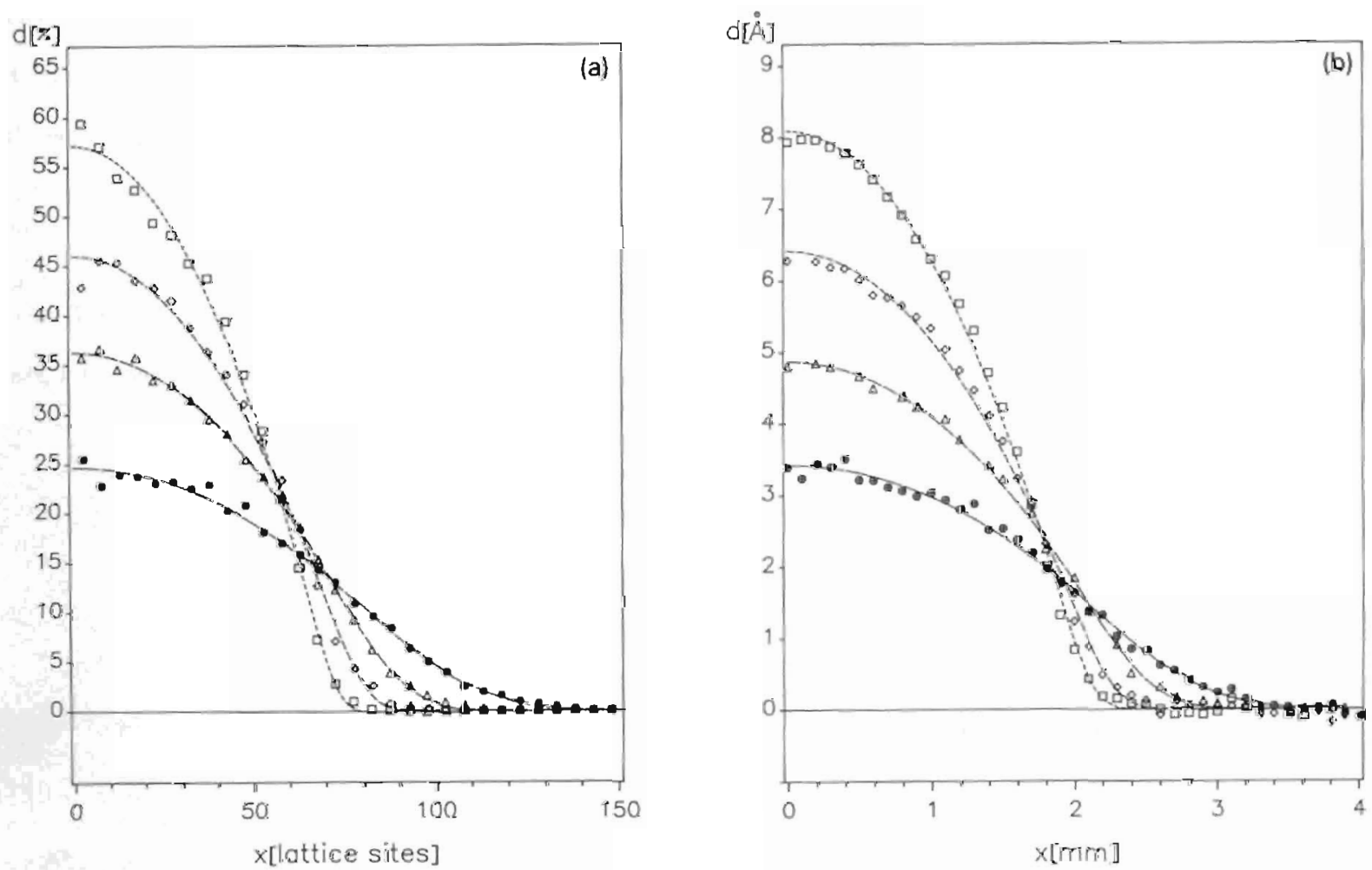

Fig. 4. (a) Droplet profiles resulting from the simulation at $2000(\square), 4000(\diamond), 8000(\Delta)$ and $18000(\oplus)$ Monte Carlo steps/monomer, $d$ is given in percent of occupied lattice sites. (b) Experimental PDMS droplet profiles $50(\square), 80(0), 150(\Delta)$ and $360(\oplus)$ minutes after deposition. The dashed lines show fits to spherical caps convoluted with a narrow Gaussian to account for the smeared out foot. 
This was confirmed by simulations with different polymer lengths. There the relative width of this Gaussian component was seen to depend on the polymer size, runs with shorter chains exhibiting a more pronounced foot. Apart from that, the runs with other chain lengths gave essentially the same results.

The droplet radii in the simulations grow with $t^{0.2}$, somcwhat faster than in the experiments which also showed indications for different growth regimes, i.e., a simple law $r \sim t^{\gamma}$ might not be applicable. This difference might be a consequence of the limited number of polymers in the simulation. Additional effects which have not been accounted for, likc pinning of molecules to surface heterogeneities, could also play a role.

To conclude we have for the first time measured the profiles of polymer droplets with submonolayer thickness and their evolution with time. These systems are experimental realisations of a two-dimensional polymer gas. The diffusion characteristics are quite different from those expected for free particle diffusion, only at very low areal polymer densities free diffusion sets in. Monte Carlo simulations of two-dimensional polymer ensembles using the bond-fluctuation method yield the same sperical cap shaped profiles as the expcriments and even reproduce the essential features of the spreading dynamics. The observed behavior thus appears to be almost exclusively due to the polymeric nature of the molecules, leading to a hindered diffusion even at submonolayer areal densities. This should be taken into account in a description of the spreading behavior of thin polymer films. The bondfluctuation method appears to be an appropriate technique to model the diffusion of polymers.

This work was supported by the SFB 306 of the Deutsche Forschungsgemeinschaft.

\section{References}

[1] P.G. de Gennes, Rev. Mod. Phys. 57 (1985) 827

[2] See, e.g.: F. Hesiot, A.M. Cazabat and N. Fraysse. J. Phys. Condens. Matter I (1989) 5793;

D. Beaglehole. J. Phys. Chem. 93 (1989) 893;

F. Heslot, N. Fraysse and A.M. Cazabat, Nature 338 (1989) 640;

F. Heslot, A.M. Cazabat, P. Levinson and N. Fraysse, Phys. Rev. Letl. 65 (1990) 599;

J. Daillant, J.J. Benattar and L. I eger, Phys. Rev. A 41 (1990) 1963, and references thurein.

[3] A.M. Cazabat, N. Fraysse, F. Heslot and P. Carles, J. Phys. Chem. 94 (1990) 7581, and references therein.

[4] R.G. Horn and J.N. Israelachvili, Macromolecules 21 (1988) 2836 .

[5] Aldrich cat. no. 31766-7, mean molecular weight $M_{w}=760$, viscosity $\eta=4.6 \times 10^{3}$ Pas, surface tension $\sigma=0.021 \mathrm{~N} / \mathrm{m}$,

[6] U. Albrucht, H. Dilger, P. Evers and P. Leiderer, in: Process Module Metrology, Control and Clustering, Eds. C.J. Davis, I.P. Herman and T.R. Turner, Proc. SPIE 1594 (1992) 344.

[7] I. Carmesin and K. Kremer, Macromolecules 21 (1988) 2819 ; J. Phys. (Paris) 51 (1990) 915 